\title{
REFERÊNCIAS HEMATOLÓGICAS E BIOQUIIMICAS EM COELHOS DOMÉSTICOS (Oryctolagus cuniculus) DA REGIÃO DE CANOAS (RS)
}

\author{
Luize Brenner ${ }^{1}$ \\ Jessica Laux ${ }^{1}$ \\ Kimberli de Oliveira Duarte ${ }^{2}$ \\ Elisandro Oliveira dos Santos ${ }^{1}$ \\ Mariangela da Costa Allgayer ${ }^{3}$
}

\begin{abstract}
RESUMO
Os coelhos domésticos (Oryctolagus cuniculus) são criados para produção de carne, lã, pele, pesquisas em laboratório e como animais de companhia. $\mathrm{O}$ objetivo do presente trabalho foi determinar os parâmetros hematológicos e bioquímicos de coelhos domésticos com raças variadas, no Hospital Veterinário da Universidade Luterana do Brasil (ULBRA/CANOASRS). Para a determinação bioquímica foram utilizados kits reagentes Labtest ${ }^{\circledR}$, analisados em espectrofotômetro BA-88 Mindray ${ }^{\circledR}$, o hemograma foi realizado em contador hematológico (Sysmex ${ }^{\circledR}$ PocH-100iV Diff) com diferenciação leucocitária por microscopia óptica. A proteína plasmática total (PPT) foi realizada por refratometria, para o fibrinogênio usou-se a técnica de precipitação por calor. Os valores bioquímicos obtidos foram: Albumina 4,0 - 5,2 $\mathrm{g} / \mathrm{dL}$, alanina aminotransferase (ALT) 42,0 - 68,0 UI/L, creatinina 1,1 - 1,3 mg/dL, gamaglutamil transferase (GGT) 21,0 - 27,0 UI/L e ureia 38,0 - 46,0 mg/dL. Os parâmetros hematológicos foram: Eritrócitos $\left(\mathrm{x} 10^{6} \mu \mathrm{L}\right) 5,4$ - 6,8; hematócrito (\%) 41 - 49; Volume Corpuscular Médio (VCM) (fL) 71 - 77; Amplitude de Distribuição dos Eritrócitos (RDW) (\%) 13,2 - 17,8; plaquetas $(\mu \mathrm{L}) 328.000$ - 544.000; PPT (g/dL) 6,5 - 7,7; fibrinogênio $(\mathrm{mg} / \mathrm{dL}) 12$ - 300; leucócitos totais $(\mu \mathrm{L}) 5.800-10.800$; heterófilos $(\mu \mathrm{L}) 1.400-4.400$; eosinófilos $(\mu \mathrm{L}) 0$ - 360; linfócitos $(\mu \mathrm{L}) 2.800$ - 6.600; monócitos $(\mu \mathrm{L}) 40$ - 800; basófilos $(\mu \mathrm{L}) 0-36$.
\end{abstract}

Palavras-chave: hemograma, lagomorfos, patologia clínica.

\section{HEMATOLOGICAL AND BIOCHEMICAL REFERENCE VALUES IN DOMESTIC RABBITS (Oryctolagus cuniculus) FROM CANOAS CITY (RS)}

\begin{abstract}
Domestic rabbits (Oryctolagus cuniculus) are bred for the production of meat, wool, for, laboratory research and as companion animals. The objective of the present work was to determine the hematological and biochemical parameters of domestic rabbits of different breeds, at the Veterinary Hospital of Universidade Luterana do Brasil (ULBRA/CANOASRS). For biochemical determination, Labtest ${ }^{\circledR}$ reagent kits were used, analyzed in a BA-88 Mindray ${ }^{\circledR}$ spectrophotometer, the blood count was performed in a hematological counter (Sysmex ${ }^{\circledR}$ PocH-100iV Diff) with leukocyte differentiation by optical microscopy. Total plasma protein (TPP) was performed by refractometry, for fibrinogen the heat precipitation technique was used. The biochemical values obtained were: Albumin $4.0-5.2 \mathrm{~g} / \mathrm{dL}$, alanine aminotransferase (ALT) $42.0-68.0 \mathrm{IU} / \mathrm{L}$, creatinine $1.1-1.3 \mathrm{mg} / \mathrm{dL}$, gammaglutamyltransferase (GGT) 21.0 - $27.0 \mathrm{IU} / \mathrm{L}$ and urea $38.0-46.0 \mathrm{mg} / \mathrm{dL}$. The hematological

\footnotetext{
${ }^{1}$ Hospital Veterinário da ULBRA - Canoas (RS) luh.brenner@gmail.com

${ }^{2}$ Residente em Patologia Clínica Veterinária. Universidade Luterana do Brasil. kimberli.duarte@gmail.com

${ }^{3}$ Docente da Faculdade de Medicina Veterinária. Universidade Luterana do Brasil. *Correspondência: mallgayer@ulbra.br
} 
parameters were: $\mathrm{RBC}\left(\mathrm{x} 10^{6} \mu \mathrm{L}\right) 5.4-6.8$; packed cell volume (\%) $41-49$; mean cell volume (MCV) (fL) 71 - 77; red blood cell distribution width (RDW) (\%) $13.2-17.8$; platelets $(\mu \mathrm{L})$ 328.000 - 544.000; TPP $(\mathrm{g} / \mathrm{dL}) 6.4$ - 7.7; fibrinogen $(\mathrm{mg} / \mathrm{dL}) 12-300$; leukocytes $(\mu \mathrm{L})$ $5.800-10.800$; heterophiles $(\mu \mathrm{L}) 1.400-4.400$; eosinophils $(\mu \mathrm{L}) 0-360$; lymphocytes $(\mu \mathrm{L})$ $2.800-6.600$; monocytes $(\mu \mathrm{L}) 40-800$; basophils $(\mu \mathrm{L}) 0-36$.

Keywords: blood count, clinical pathology, lagomorphs.

\section{REFERENCIAS HEMATOLÓGICAS Y BIOQUÍMICAS EN CONEJOS DOMÉSTICOS (Oryctolagus cuniculus) DE LA REGIÓN DE CANOAS (RS)}

\section{RESUMEN}

Los conejos domésticos (Oryctolagus cuniculus) se crían para carne, lana, producción de pieles, investigación de laboratorio y como animales de compañía. El objetivo del presente trabajo era determinar los parámetros hematológicos y bioquímicos de conejos domésticos de diferentes razas, en el Hospital Veterinario de la Universidade Luterana do Brasil (ULBRA/CANOAS - RS). Para la determinación bioquímica se utilizaron kits de reactivos Labtest $^{\circledR}$, analizados en un espectrofotómetro BA-88 Mindray ${ }^{\circledR}$, el hemograma se realizó en un contador hematológico (Sysmex ${ }^{\circledR}$ PocH-100iV Diff) con diferenciación leucocitaria por microscopía óptica. La proteína plasmática total (PPT) se realizó mediante refractometría, para el fibrinógeno se utilizó la técnica de precipitación por calor. Los valores bioquímicos obtenidos fueron: Albúmina 4,0 - 5,2 g/dL, alanina aminotransferasa (ALT) 42,0 - 68,0 UI/L, creatinina $1,1-1,3 \mathrm{mg} / \mathrm{dL}$, gamma-glutamil transferasa (GGT) $21,0-27,0 \mathrm{UI} / \mathrm{L}$ y urea 38,0 $46,0 \mathrm{mg} / \mathrm{dL}$. Los parámetros hematológicos fueron: Eritrocitos $\left(\times 10^{6} \mu \mathrm{L}\right) 5,4-6,8$; hematocrito (\%) 41 - 49; Volumen corpuscular medio (MCV) (fL) 71 - 77; Rango de distribución de eritrocitos (RDW) (\%) 13,2 - 17,8; plaquetas $(\mu \mathrm{L}) 328.000$ - 544.000; PPT $(\mathrm{g} / \mathrm{dL})$ 6,5 - 7,7; fibrinógeno $(\mathrm{mg} / \mathrm{dL}) 12$ - 300; leucocitos totales $(\mu \mathrm{L})$ 5,800 - 10,800; heterófilos $(\mu \mathrm{L}) 1.400$ - 4.400; eosinófilos $(\mu \mathrm{L}) 0$ - 360; linfocitos $(\mu \mathrm{L}) 2.800$ - 6.600; monocitos $(\mu \mathrm{L}) 40-800$; basófilos $(\mu \mathrm{L}) 0-36$.

Palabras clave: hemograma, lagomorfos, patología clínica.

\section{INTRODUÇÃO}

Um dos métodos mais utilizados para avaliação complementar e monitoramento do estado de saúde geral em qualquer espécie animal é a avaliação dos valores hematológicos e bioquímicos, por serem bons indicadores fisiológicos, patológicos e do estado nutricional do paciente (1). Devido às diferentes condições ambientais, climáticas, manejo e os métodos laboratoriais utilizados as análises podem apresentar variações evidentes. Por este motivo, cada laboratório deve possuir a sua avaliação destes valores, decorrente do método em que cada um utiliza $(1,2)$.

Dentro deste contexto, o objetivo do presente trabalho foi o estabelecimento de intervalos de referência dos parâmetros hematológicos e bioquímicos de coelhos para o Laboratório de Patologia Clínica do Hospital Veterinário da Universidade Luterana do Brasil em Canoas (RS).

\section{MATERIAIS E MÉTODOS}

Brenner L, Laux J, Duarte KO, Santos EO, Allgayer MC. Referências hematológicas e bioquímicas em coelhos domésticos (Oryctolagus cuniculus) da região de Canoas (RS). Vet. e Zootec. 2021; v28: 001-007. 
Para o estudo dos parâmetros hematológicos foram utilizados 32 animais no total, 21 fêmeas e 11 machos. Enquanto para a análise sérica foram utilizados 21 animais, dentre eles, 13 fêmeas e 8 machos. Os animais eram das raças Mini Lop, Mini Lion Head, Netherland Dwarf, Fuzzy Lop, Jersey Wooly, Siames Esmoquin, Teddy Dwerg e sem raça definida.

Os animais coletados eram oriundos de tutores e da rotina do Hospital Veterinário da ULBRA. Para avaliação da sanidade destes animais, foram realizados exames clínico e parasitológico previamente à coleta. Todos os animais estavam em idade reprodutiva (entre 4 meses e 8 anos) e a alimentação era constituída de ração comercial, feno e folhas verdes. Apresentavam-se clinicamente sadios e não utilizavam nenhum medicamento no momento da coleta.

Foram realizadas duas coletas no mês de novembro de 2019, entre 09h00min e $12 \mathrm{~h} 00 \mathrm{~min}$. O sangue foi coletado por punção da veia safena lateral, em volumes que variaram entre 0,5 e $2 \mathrm{~mL}$ de cada animal e acondicionado em tubos com EDTA e tubos sem anticoagulante contendo ativador de coágulo. As amostras foram armazenadas dentro de um isopor com gelo e encaminhadas até o Laboratório de Patologia Clínica do HV-ULBRA.

As amostras com EDTA foram homogeneizadas durante 5 minutos, após foi confeccionado o esfregaço sanguíneo corados com Panótico Rápido ${ }^{\circledR}$. Para a determinação da PPT, foi realizado o preenchimento do capilar e centrifugação por 5 minutos para obtenção do plasma e leitura no refratômetro.

As análises hematológicas foram realizadas por meio de contador hematológico veterinário (Sysmex ${ }^{\circledR}$ PocH-100iV Diff) calibrado para a espécie. Sendo determinado o hematócrito, número total de eritrócitos, volume globular médio (VCM), RDW e número total de leucócitos; com diferencial leucocitário e contagem de plaquetas, realizados por microscopia óptica.

Para avaliar a concentração do fibrinogênio foi realizada a técnica de precipitação por calor em banho-maria na temperatura de $56^{\circ} \mathrm{C}$ por 3 minutos, logo após as amostras foram centrifugadas por 5 minutos para leitura no refratômetro. $\mathrm{O}$ valor do fibrinogênio plasmático foi calculado pela subtração do índice de refração do plasma não incubado pelo índice de refração do plasma incubado, expresso em $\mathrm{mg} / \mathrm{dL}$.

As amostras sem anticoagulantes foram deixadas à temperatura ambiente para a formação do coágulo, e posteriormente todas as amostras foram centrifugadas na centrífuga para tubos (Quimis $\left.{ }^{\circledR}\right)$, com a rotação de $5.000 \mathrm{rpm}$ por 10 minutos. Os soros e plasmas obtidos foram separados, acondicionados em Eppendorf de $1,5 \mathrm{~mL}$ e congelados a $-20^{\circ} \mathrm{C}$ para posterior leitura.

Em março de 2020, as amostras foram descongeladas e processadas. As amostras de plasma foram utilizadas para mensurar ALT, GGT, ureia e creatinina, a albumina foi mensurada utilizando soro. Para a realização dos exames foram utilizados kits comerciais $\left(\right.$ Labtest $^{\mathbb{B}}$ ), a leitura foi realizada em espectrofotômetro BA-88 Mindray ${ }^{\circledR}$.

A análise estatística das amostras foi realizada utilizando-se os programas SAS (Statistical Analysis System), e o teste de $T$ Student para determinar as diferenças significativas entre as médias dos grupos analisados, e o intervalo de confiança usando a distribuição $\mathrm{T}$ para amostras com $\mathrm{n} \leq 30$. Esta análise foi feita levando em consideração uma confiança de $95 \%$, utilizando assim um ponto de corte para um p-valor igual a 5\%. Ou seja, para valores de p menores que 0,05 , pode-se afirmar que a hipótese é rejeitada com nível de confiança de $95 \%$.

O projeto de pesquisa foi aprovado pelo comitê de ética da Universidade Luterana do Brasil, sob o número 587/2019.

\section{RESULTADOS}

Brenner L, Laux J, Duarte KO, Santos EO, Allgayer MC. Referências hematológicas e bioquímicas em coelhos domésticos (Oryctolagus cuniculus) da região de Canoas (RS). Vet. e Zootec. 2021; v28: 001-007. 
Os resultados obtidos para enzimas hepatobiliares, compostos nitrogenados e albumina em coelhos miniatura hígidos, suas médias e desvios padrões e o intervalo de referência estão apresentados na Tabela 1. Ao analisar os resultados das fêmeas e machos, não se evidenciou diferença significativa no perfil bioquímico em relação ao sexo.

Tabela 1. Médias, margens de erro e intervalo de referência para parâmetros bioquímicos de coelhos de estimação adultos e saudáveis

\begin{tabular}{ccccc}
\hline Parâmetros & n & Média & Desvio Padrão & Intervalo de referência \\
\hline Albumina (g/dL) & 8 & 4,6 & 0,6 & $4,0-5,2$ \\
\hline ALT (UI/L) & 10 & 55,0 & 13,0 & $42,0-68,0$ \\
\hline Creatinina (mg/dL) & 19 & 1,2 & 0,10 & $1,1-1,3$ \\
\hline GGT (UI/L) & 18 & 24,0 & 3,0 & $21,0-27,0$ \\
\hline Ureia (mg/dL) & 21 & 42,0 & 4,0 & $38,0-46,0$ \\
\hline
\end{tabular}

Os valores médios obtidos nos parâmetros hematológicos dos coelhos domésticos estão sendo representados na Tabela 2. Houve diferença estatística em relação ao sexo apenas no número de eritrócitos $(\mathrm{p}<0,02)$ e hematócrito $(\mathrm{p}<0,01)$. As fêmeas apresentaram valores de

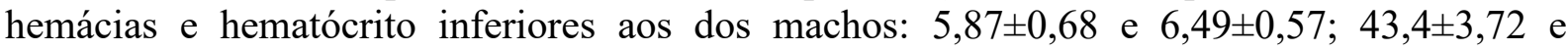
$47,11 \pm 3,48$, respectivamente.

Tabela 2. Média e desvio padrão obtidos no hemograma, PPT, fibrinogênio de coelhos de estimação

\begin{tabular}{cccc}
\hline Parâmetros $(\mathbf{n}=\mathbf{3 2})$ & Média & Desvio padrão & Intervalo de referência \\
\hline Eritrócitos $\left(\times 10^{6} \mu \mathrm{L}\right)$ & 6,1 & 0,7 & $5,4-6,8$ \\
\hline Hematócrito $(\%)$ & 45 & 4,0 & $41-49$ \\
\hline VCM $(\mathrm{fL})$ & 74 & 3,0 & $71-77$ \\
\hline RDW CV $(\%)$ & 15,5 & 2,3 & $13,2-17,8$ \\
\hline Plaquetas $\left(\times 10^{3} \mu \mathrm{L}\right)$ & 436.000 & 108.000 & $328.000-544.000$ \\
\hline PPT $(\mathrm{g} / \mathrm{dL})$ & 7,1 & 0,6 & $6,5-7,7$ \\
\hline Fibrinogênio $(\mathrm{mg} / \mathrm{dL})$ & 156 & 144 & $12-300$ \\
\hline Leucócitos totais $(\mu \mathrm{L})$ & 8.300 & 2.500 & $5.800-10.800$ \\
\hline Heterófilos $(\mu \mathrm{L})$ & 2.900 & 1.500 & $1.400-4.400$ \\
\hline Eosinófilos $(\mu \mathrm{L})$ & 178 & 179 & $0-360$ \\
\hline Linfócitos $(\mu \mathrm{L})$ & 4.700 & 1.900 & $2.800-6.600$ \\
\hline Monócitos $(\mu \mathrm{L})$ & 420 & 380 & $0-800$ \\
\hline Basófilos $(\mu \mathrm{L})$ & 10 & 26 & $0-36$ \\
\hline
\end{tabular}

\section{DISCUSSÃO}

Para evitar uma variação nos parâmetros bioquímicos analisados, as coletas foram realizadas entre $09 \mathrm{~h} 00 \mathrm{~min}$ e $12 \mathrm{~h} 00 \mathrm{~min}$. Campbell (3) relata que a ureia é o principal parâmetro em que a concentração plasmática em coelhos pode ser influenciada pela hora da coleta, notando-se uma concentração máxima entre as $16 \mathrm{~h} 00 \mathrm{~min}$ e $20 \mathrm{~h} 00 \mathrm{~min}$. Melillo (4) também afirma essa relação com o horário da coleta, relatando que a concentração de ureia tende a aumentar no final do dia. Essa variação também pode ser vista nos parâmetros hematológicos, conforme Moore et al. (5), o número de neutrófilos circulantes é mais alto no 
início da manhã e mais baixo no final da tarde e à noite. O horário das coletas evitou essa variação, assim como a contenção dos animais, que foi realizada pelos tutores e o médico veterinário responsável, minimizando o estresse dos animais e alterações no comportamento de neutrófilos.

É possível que as fêmeas de coelhos apresentem uma concentração plasmática de albumina maior do que os machos (3) e machos apresentem valores maiores de ureia (6). No entanto, não houve diferença significativa entre estes valores no presente estudo.

Em relação aos parâmetros hematológicos, verificamos que apenas o número de eritrócitos e hematócrito apresentaram diferença significativa em relação ao sexo. Segundo Kerr (7), os animais machos tendem a possuir um maior número de eritrócitos devido aos efeitos de hormônios andrógenos que estimulam a eritropoiese. $\mathrm{O}$ aumento do cortisol na circulação sanguínea pode levar ao aumento do VCM, assim como amostras velhas e auto aglutinação. Porém, todas as amostras do presente estudo foram processadas dentro de 3 horas após a chegada, descartando essa hipótese, assim como não houve diferença significativa nos valores de VCM obtidos, na comparação entre machos e fêmeas.

Os valores obtidos para ALT neste estudo (42,0 - 68,0 UI/L) se encaixam nos valores de referência indicados na literatura comparada de Quinton (8), Hernandes-Divers (9), Pessoa (10) e Campbell (3) e na publicação de Melillo (4).

Segundo Hernandes-Divers (9), o valor de referência para albumina em coelhos é de 2,5 a 4,5 g/dL. Os valores obtidos neste trabalho $(4,0-5,2 \mathrm{~g} / \mathrm{dL})$ se comparam, de forma geral, com os intervalos estabelecidos na literatura onde Pessoa (10) e Melillo (4) indicam um intervalo de referência máximo mais elevado, entre 2,7 a $5,0 \mathrm{~g} / \mathrm{dL}$. Özkan et al. (11) publicaram intervalos de referência diferentes para machos e fêmeas, sendo $2,7-4,3 \mathrm{~g} / \mathrm{dl} \mathrm{e}$ $2,3-3,5 \mathrm{~g} / \mathrm{dl}$, respectivamente.

Segundo Melillo (4) níveis de ureia em coelhos dependem do ritmo circadiano, onde o pico é observado no final da tarde e início da noite. Outros fatores incluem a quantidade e qualidade de proteínas na dieta, estado nutricional, função fígado, absorção intestinal, atividade urease da microbiota cecal e estado de hidratação. Intervalos de referência para a ureia foram publicados por Melillo (4), Elamin (6) e Emanuelli et al. (12), sendo 20 - 45 $\mathrm{mg} / \mathrm{dL}, 20,40-59,40 \mathrm{mg} / \mathrm{dL}$ e 9,24 - 66,06 mg/dL, respectivamente. Nota-se que estes valores são próximos aos encontrados neste trabalho.

Os valores de referência para a creatinina obtidos por Melillo (4), Elamin (6) e Özkan et al. (11) foram de $0,5-2,5 \mathrm{mg} / \mathrm{dL}, 1,69-2,81 \mathrm{mg} / \mathrm{dL}$, e $1,81-2,52 \mathrm{mg} / \mathrm{dL}$, respectivamente. Torna-se notório que os valores de referência mínimos obtidos por Elamin (6) e Özkan et al. (11) são mais altos que as demais publicações. Emanuelli et al. (12) publicaram valores máximos de referência para a creatinina mais baixos que os demais, $0,51-1,53 \mathrm{mg} / \mathrm{dL}$. Os valores obtidos no presente estudo $(1,1-1,3 \mathrm{mg} / \mathrm{dL})$ estão entre a margem de valores citados na literatura por Hoefer (13), Quinton (8) e Hernandes-Divers (9) e dos encontrados por Melillo (4) e Emanuelli et al. (12).

Dentre as análises realizadas neste trabalho, os valores obtidos de GGT (21,0 - 27,0 UI/L) são mais altos quando comparados com os obtidos por Pessoa (10) e Campbell (3). Os valores de referência publicado por Emanuelli et al. (12) $(2,0-15,0 \mathrm{UI} / \mathrm{L})$ também se apresentaram superiores aos observados na literatura no qual destaca a importância de valores de referência para bioquímica sérica em coelhos, devido a variabilidade dos resultados encontrados por diversos autores.

Os valores hematológicos obtidos neste trabalho, ao serem comparados com os intervalos estabelecidos por Leineweber et al. (14) e Gallego (15), mostram-se próximos e alguns números dentro das referências relatados por estes autores. Na presente pesquisa, os resultados obtidos não apresentaram diferenças relevantes provavelmente devido à mesma metodologia empregada e ao estado sanitário dos animais amostrados. 
Murata et al. (16) e Valera e Milán (17) relatam que o fibrinogênio é uma proteína de fase aguda evidenciada no início de processos inflamatórios agudos e utilizada com frequência na avaliação inicial de uma resposta mais sensível e precoce em relação a resposta leucocitária em herbívoros. Este trabalho sugere que a dosagem de fibrinogênio seja feita em lagomorfos sempre que haja uma suspeita de processos inflamatórios pelo médico veterinário responsável, como uma ferramenta de auxílio diagnóstico, já que assim como nas outras espécies herbívoras, há uma relação neutrófilo/linfócito invertida, sendo a resposta de neutrófilos evidenciada tardiamente no leucograma. Na literatura consultada não foram encontrados valores de referência para o fibrinogênio em coelhos.

\section{CONCLUSÕES}

Os resultados obtidos neste estudo podem servir como referência na avaliação clínica destes animais, auxiliando o médico veterinário quanto às alterações hematológicas encontradas nas diferentes patologias, já que a literatura fornece poucos dados sobre valores de referência em coelhos criados como animais de estimação.

\section{REFERÊNCIAS}

1. Andrade A, Pinto SC, Oliveira RS. Animais de laboratório: criação e experimentação. Rio de Janeiro: Editora Fiocruz; 2002.

2. Bush BM. Interpretação de resultados laboratoriais para clínicos de pequenos animais. São Paulo: Roca; 2004.

3. Campbell TW. Bioquímica clínica dos mamíferos: animais de laboratório e espécies variadas. In: Thrall MA, Weiser G, Allison RW, Campbell TW. Hematologia e bioquímica clínica veterinária. 2a ed. Rio de Janeiro: Roca; 2015. Cap. 34, p. 1209-32.

4. Melillo A. Topics in medicine and surgery: rabbit clinical pathology. J Exot Pet Med. 2007;16(3):135-45. doi: 10.1053/j.jepm.2007.06.002.

5. Moore DM, Zimmerman K, Smith SA. Hematological assessment in pet rabbits: blood sample collection and blood cell identification. Vet Clin Exot Anim Pract. 2015;18(1): 919. doi: 10.1016/j.cvex.2014.09.003.

6. Elamin KM. Age and sex effects on blood biochemical profile of local rabbits in Sudan. Wayamba J Anim Sci. 2013;5(1):548-53.

7. Kerr MG. Exames laboratoriais em medicina veterinária: bioquímica clínica e hematológica. 2a ed. São Paulo: Roca; 2003.

8. Quinton JF. Novos animais de estimação. São Paulo: Roca; 2005.

9. Hernandes-Divers SJ. Coelhos. In: Carpenter JW. Formulário de animais exóticos. 3a ed. São Paulo: Medvet; 2010. Cap. 10, p. 690-739.

10. Pessoa CA. Lagomorpha (coelho, lebre e tapiti). In: Cubas ZS, Silva JCR, Catão-Dias JL. Tratado de animais selvagens. 2a ed. São Paulo: Roca; 2014. Cap. 56, p. 1209-37. 
11. Özkan C, Kaya A, Akgül Y. Normal values of haematological and some biochemical parameters in serum and urine of New Zealand white rabbits. World Rabbit Sci. 2012;20(4):253-9. doi: 10.4995/wrs.2012.1229.

12. Emanuelli MP, Lopes STA, Maciel RM, Garmatz BC, Tavares MO. Concentração sérica de fosfatase alcalina, gama-glutamil transferase, ureia e creatinina em coelhos (Oryctolagus cuniculus). Cienc Anim Bras. 2008;9(1):251-5.

13. Hoefer HL. Rabbit and ferret renal disease diagnosis. In: Fudge AM. Laboratory medicine: avian and exotic pets. Philadelphia: WB Saunders Company; 2000. Cap. 38, p. $311-8$.

14. Leineweber C, Muller E, Marschang RE. Blood reference intervals for rabbits (Oryctolagus cuniculus) from routine diagnostic sample. Tierarztl Prax Ausg K Kleintiere Heimtiere. 2018;46(6):393-8. doi: 10.1055/s-0038-1677403.

15. Gallego M. Laboratory reference intervals for systolic blood pressure, rectal temperature, hematology, biochemistry and venous blood gas and electrolytes in healthy pet rabbits. Open Vet J. 2017;7(1):203-7. doi: 10.4314/ovj.v7i3.1.

16. Murata H, Shimada N, Yoshioka M. Current research on acute phase proteins in veterinary diagnosis: an overview. Vet J. 2004;167(1):28-40. doi: 10.1016/S10900233(03)00119-9.

17. Valera RC, Milán JP. Utilidad del hemograma em la clínica equina. Equinus Med Cir Equina. 2006;14(1):11-27.

Recebido em: 14/09/2021

Aceito em: 08/12/2021 